\title{
Observation of the Presuperfluid Regime in a Two-Dimensional Bose Gas
}

\author{
S. Tung, G. Lamporesi, D. Lobser, L. Xia, and E. A. Cornell* \\ JILA, National Institute of Standards and Technology and University of Colorado, \\ and Department of Physics, University of Colorado, Boulder, Colorado 80309-0440, USA
}

(Dated: September 18, 2018)

\begin{abstract}
In complementary images of coordinate-space and momentum-space density in a trapped 2D Bose gas, we observe the emergence of presuperfluid behavior. As phase-space density $\rho$ increases toward degenerate values, we observe a gradual divergence of the compressibility $\kappa$ from the value predicted by a bare-atom model, $\kappa_{b a} . \kappa / \kappa_{b a}$ grows to 1.7 before $\rho$ reaches the value for which we observe the sudden emergence of a spike at $p=0$ in momentum space. Momentum-space images are acquired by means of a $2 \mathrm{D}$ focusing technique. Our data represent the first observation of non-mean-field physics in the presuperfluid but degenerate 2D Bose gas.

PACS numbers: 05.30.Jp, 67.10.Ba, 67.85.-d
\end{abstract}

Because of the enhanced role of fluctuations in lowdimensional systems [1], a two-dimensional (2D) Bose gas at nonzero temperature does not have long-range phase coherence. In a homogeneous system there can be at best only a quasicondensate, no true Bose-Einstein condensation (BEC). Under the combined effect of interactions and quantum degeneracy, however, there is nonetheless a phase transition known as Berezinskii-KosterlitzThouless (BKT) associated with the unbinding of vortex pairs [2]. Below the critical temperature $T_{\mathrm{BKT}}$, the system is superfluid.

Experiments in 2D atomic gases [3-6] are usually conducted in the presence of an inhomogeneous trapping potential. In the complete absence of interactions, the confining potential can resurrect a traditional BEC [7], but for realistic experimental parameters, interatomic interactions tend to suppress BEC by smoothing out the spatial profile [3- $[6,[\underline{8},[9]$ of the mean density to the point where the sample can be understood as a collection of locally uniform spatial regions, each of which is characterized by a particular local density and thus a particular local value of $T_{\mathrm{BKT}}$. Although these local regions may be too small to test in detail the coherence-related predictions of BKT theory, qualitative effects have been observed in experiment [3, 6].

Our particular interest is in the region just to the warm side of $T_{\mathrm{BKT}}$. In an earlier experiment on bosons trapped in a $2 \mathrm{D}$ optical lattice, we observed a proliferation of vortices as we warmed through the discrete-case equivalent of $T_{\mathrm{BKT}}$ 10]. But in that experiment a great many mesoscopic condensates were present, one at each lattice site, on both sides of the BKT transition, because they had condensed at a $T_{\mathrm{BEC}}$ distinct from and well above $T_{\mathrm{BKT}}$. For the continuous case, in contrast, there is no corresponding second transition temperature above $T_{\mathrm{BKT}}$. But if the cooling gas has by $T_{\mathrm{BKT}}$ already become a medium that can support vortices, whether bound or not, then heuristically we see that it must have continuously evolved from a fully fluctuating nondegenerate gas into a sort of presuperfluid with suppressed density fluctua- tions [11]. Theory [8, 11 15] validates this intuition, and experiments [5] have in turn been consistent with predictions of that theory. Up until now, however, experiments have not been directly sensitive to the properties of the presuperfluid, $T \gtrsim T_{\mathrm{BKT}}$ gas. The goal of the present work is to provide a minimal-assumption, first empirical look at this exotic regime. We emphasize key features of this approach: 1. Our line of sight is along the axis of tight confinement: we do not need to do a deconvolution of our images to get the 2D density distribution. Steps are taken to minimize systematic errors in density measurements. 2. We analyze our in situ images to extract the local compressibility, a quantity directly sensitive to local microscopic physics. 3 . We use a $2 \mathrm{D}$ focusing technique to record high-resolution 2D momentum-space images complementary to the coordinate-space images. We make corresponding inferences about nonlocal coherence. 4. We use a simple but robust "bare-atom" model to correct the observed density for the presence of a small population in excited states in the tight confinement direction, and to determine a bare-atom compressibility with which to compare our observations.

Experimentally, we create a stack of well-isolated quasi-2D layers by superimposing a one-dimensional, blue-detuned optical lattice with lattice spacing $3.8 \mu \mathrm{m}$ onto a magnetically trapped, evaporatively cooled cloud of Rb-87 atoms. Within each layer, approximately $6.2 \times 10^{5}$ atoms feel a harmonic potential characterized by frequencies $\left(\omega_{r}, \omega_{z}\right)=2 \pi(10,1400) \mathrm{Hz}$. The characteristic dimensionless 2D interaction strength is $\tilde{g}=$ $\sqrt{8 \pi}\left(a_{s} / a_{h o}\right)=0.093$, where $a_{s}$ is the 3D scattering length and $a_{h o}$ is the $\hat{z}$ harmonic-oscillator length [16]. The atoms are allowed to equilibrate in their 2D geometry before probing occurs. Right before probing the resulting coordinate- or momentum-space distribution, we apply a microwave pulse together with a transient magnetic field to pump atoms in the central layer into another hyperfine state, resonant with the probe light. The strength of the microwave selection pulse is adjusted to keep the peak optical density of the imaged fraction 


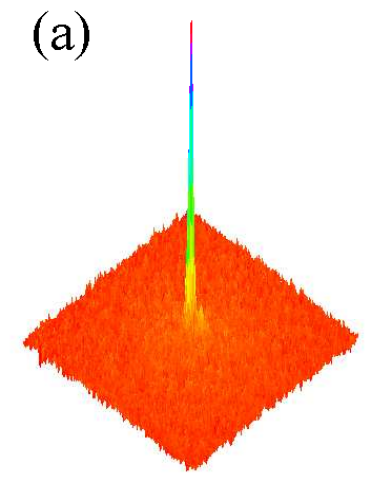

(b)

FIG. 1. (a) A trap-focused, momentum-space image. (b) An in-situ, coordinate-space image. The corresponding azimuthal averages are shown in Figs. 2(g) and 2(c).

within the linear dynamic range of our imaging.

Our momentum-space imaging makes use of a focusing technique which is an extension to $2 \mathrm{D}$ of a procedure developed for imaging 1D momentum distributions [17, 18]. It yields a much cleaner separation of momentum and coordinate-space distributions than is obtained in earlier experiments [4, 6]. After selecting a single layer, we turn off the optical lattice and let the layer expand into a purely magnetic trap with frequencies $\left(\omega_{r}, \omega_{z}\right)=2 \pi(5.2,10.4) \mathrm{Hz}$. Because of the $140: 1$ aspect ratio of the cloud, the expansion is initially purely axial, very rapidly reducing the $3 \mathrm{D}$ density while not affecting the in-plane coordinate- or momentum-space distributions. After this near-instantaneous suppression of the repulsive atom-atom interactions, each atom undergoes free harmonic motion in the $x-y$ plane. After a dwell time $t=\frac{1}{4} \frac{2 \pi}{\omega_{r}}$, just as the initial 2D momentum- and coordinate-space distributions have swapped, we take an absorption image [Fig. 1(a)]. Scaling the spatial coordinate by $m \omega_{r}$ yields the momentum-space distribution that existed just as the probe sequence began. We take azimuthal averages of the absorption images before plotting and fitting the data.

For coordinate-space images the procedure is similar, but after the lattice turn-off, we wait only $1 \mathrm{~ms}$ before taking the absorption image [Fig. 1(b)]. The 2D density remains essentially frozen while the $3 \mathrm{D}$ density - and related imaging artifacts [5] - are much reduced.

To extract a signal for many-body physics from our data, we compare our data to a fully fluctuating, bareatom model, in essence the Paris group's mean-field, Hartree-Fock, local-density model [9]. The mean occupation of a single-atom state $k$ is given by the BoseEinstein distribution, $N_{k}=\frac{1}{e^{\left(E_{k}-\mu\right) / k_{B} T}-1}$, where $E_{k}$ is the energy of the state. For our system, $k_{B} T \gg \hbar \omega_{r}$, but $k_{B} T \sim \hbar \omega_{z}$. We treat the atomic motion semiclassically in the in-plane direction, while preserving discrete harmonic-oscillator levels in the $\hat{z}$ direction. The 2D coordinate-space density in the $j$ th axial level is

$$
n_{j}(\vec{r})=\frac{1}{h^{2}} \iint \mathrm{d}^{2} \vec{p} \frac{1}{e^{\left[\varepsilon(\vec{p})+\theta_{j}(\vec{r})-\mu_{j}(\vec{r})\right] / k_{B} T}-1},
$$

where the free particle dispersion $\varepsilon(\vec{p})=\frac{p^{2}}{2 m}$. The local chemical potential for the $j$ th level is given by

$$
\begin{aligned}
\mu_{j}(\vec{r}) & =\mu^{g l o b a l}-\frac{1}{2} m \omega_{r}^{2} r^{2}-j \hbar \omega_{z} \\
& -\sum_{l \neq j} 2\left(\frac{4 \pi \hbar^{2}}{m} a_{s} f_{j l} n_{l}(\vec{r})\right),
\end{aligned}
$$

whereas the intralevel interaction energy is

$$
\theta_{j}(\vec{r})=2\left(4 \pi \hbar^{2} a_{s} / m\right) f_{j j} n_{j} .
$$

The relevant mean-field interaction energies depend on $f_{j l}$ which are the normalized density overlap integrals over the axial dimension between densities associated with axial quantum states $j$ and $l$. Interaction energies are comfortably less than the axial spacing $\hbar \omega_{z}$, justifying our treating the axial wave functions as frozen. We define a quantity $u_{00} \equiv\left(4 \pi \hbar^{2} a_{s} / m\right) f_{00}$, such that we can write $\theta_{0}(\vec{r})=2 u_{00} n_{0}=2\left(\hbar^{2} \tilde{g} / m\right) n_{0}(\vec{r})$. Evaluating the integral in Eq. (11), we get

$$
n_{j}(\vec{r})=-\ln \left(1-e^{-\left[\theta_{j}(\vec{r})-\mu_{j}(\vec{r})\right] / k_{B} T}\right) / \lambda_{d b}^{2}
$$

where the de Broglie wavelength $\lambda_{d b}=\sqrt{2 \pi \hbar^{2} / m k_{B} T}$. For any given value of $\mu^{\text {global }}$ and $T, n_{j}$ are determined self-consistently. For $k_{B} T \lesssim \hbar \omega_{z}$, the model converges in just a few iterations.

The bare-atom model is a no-condensate model from which all the many-body effects associated with degenerate bosons has been intentionally omitted: the additional factor of 2 in front of the parentheses in Eq. (2) and (3) arises from an implicit assumption that the second-order correlation function at zero distance is 2 , as it would be for fully fluctuating, nondegenerate ideal bosons, and not 1 , as for a 3D BEC. Furthermore, $\varepsilon(\vec{p})=p^{2} / 2 m$ is the dispersion relation for independent atoms moving in a mean-field potential. There are no collective excitations such as phonons.

All the same, the bare-atom model should do very well where phase-space density $\rho_{j} \equiv n_{j} \lambda_{d b}^{2}<1$, true for $j>$ 0 in our system. As for the calculated value of $n_{0}(\vec{r})$, this will begin to fail for $\rho_{0} \gtrsim 3.5$, but a comparison observations with the naive, bare-atom $n_{0}$ will allow us to quantify the telltale discrepancy.

An analysis of a coordinate-space image proceeds as follows. We measure the integrated density in the $z$ direction with contributions from all populated excited axial levels. $n_{\text {meas }}(\vec{r})=\sum_{j} n_{j}(\vec{r})$, but the interesting physics lies in $n_{0}(\vec{r})$. We compare the results of the bare-atom model to observed $n_{\text {meas }}$ and fit the parameters $T$ and $\mu^{\text {global }}$ to the low phase-space (hence wellunderstood) regions of the cloud. With the chemical potential and temperature obtained from the fit, we can use 

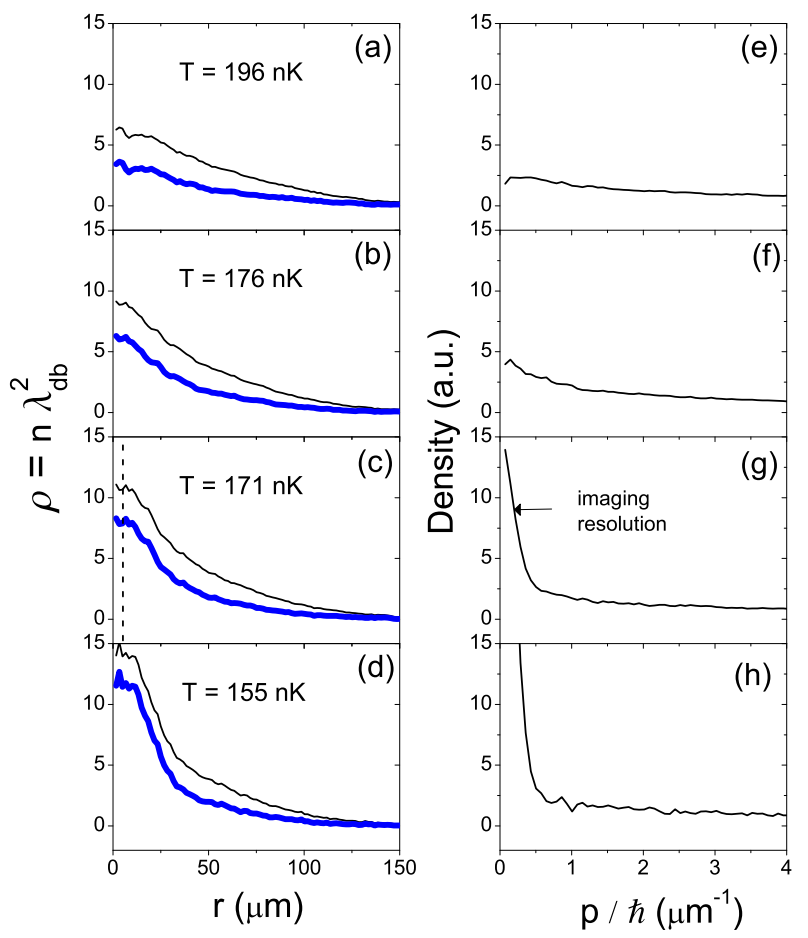

FIG. 2. (a)-(d) Coordinate-space distributions and (e)-(h) corresponding momentum distributions. Two distributions in the same row are taken under near-identical conditions. The thin black curves give the azimuthal averages of $n_{\text {meas }}$ from the raw images. The thick blue curves in the coordinatespace distributions are the ground-state distribution $n_{0}$ after correcting for $n_{k>0}$. The spike in momentum space that first appears in (g) has no corresponding dramatic change in coordinate space (c). The vertical dotted line in (c) represents the inverse of the momentum resolution limit indicated in $(\mathrm{g})$ and is thus a lower limit on the spatial extent of the coherence of the population of low-p (high-coherence) atoms represented by the area (about $1.4 \%$ of total) under the spike in $(\mathrm{g})$.

Eq. (4) to evaluate the excited state distributions. Then we numerically find a self-consistent solution to get $n_{k}$ $(k>0)$, with the constraint $n_{0}=n_{\text {meas }}-\sum_{k>0} n_{k}$; see Figs. 2(a)-2(d).

Once the corrected ground-state distribution $n_{0}$ is extracted from $n_{\text {meas }}$, we calculate the scaled (by $n^{2}$ ) isothermal compressibility $\kappa$ at each imaging pixel

$$
\kappa=d n_{0} / d \mu_{0}=\left(d n_{0} / d r\right)\left(d \mu_{0} / d r\right)^{-1} .
$$

Although $\mu^{\text {global }}$ is not a quantity we can know with great accuracy in a model-independent way, $d \mu_{0} / d r \approx$ $-d\left(\frac{1}{2} m \omega_{r}^{2} r^{2}\right) / d r=-m \omega_{r}^{2} r$ is known quite precisely, as the contributions to $\mu_{0}$ arising from the mean field of axially excited atoms are small and correctable. $d n_{0} / d r$ is determined from our coordinate-space images. Equation (5) then gives $\kappa$ at each discrete radius in an image. We plot the result vs the local phase-space density $\rho_{0}=$ $n_{0} \lambda_{d b}^{2}$ in Fig. 3. We compare $\kappa$ to the value $\kappa_{b a}$ that the bare-atom model would predict at the same density. For an observed value of $n_{0}$, we numerically solve the bareatom prediction $n_{0}=-\ln \left[1-\exp \left(\mu_{0}-\theta_{0}\left(n_{0}\right)\right) / k_{B} T\right] / \lambda_{d b}^{2}$ for $\mu_{0}$, and determine how $n_{0}$ changes for small changes in $\mu_{0}$, and thus extract $\kappa_{b a}$ (Fig. 3).

As a test of the local-density approximation that is central to our analysis, we determine $\kappa$ using images from two very different classes of samples: clouds with $T=$ $171 \mathrm{nK}$ and central $\rho_{0}$ of about 9 [Fig. 3(a)], and clouds with $T=128 \mathrm{nK}$ and central $\rho_{0}$ of about 30 [Fig. 33(b)]. The shape of $\kappa(\rho)$ is the same and, in particular, the value of $\rho_{0}$ for which the extracted value of $\kappa$ becomes distinguishable from $\kappa_{b a}$ is in both cases about 4 .

A note on our preferred method of fixing of $\alpha$, the calibration scale factor that relates $n_{\text {meas }}(\vec{r})$ to the observed optical density profile: we considered (i) an error-prone calculation based on optical absorption cross section and (ii) a model-dependent (even unto logical circularity) fitting of $\alpha$ in the image analysis but settled finally on (iii) interleaving our data runs with auxiliary measurements of very low $T$ clouds in which the atoms are in a near-pure Thomas-Fermi inverted parabola with negligible noncondensed wings. In this limit, we assume $\mu^{g l o b a l}=u_{00} n_{0}(0)$ and thus fix $\alpha$. This assumption means that our measured values of $\kappa$, scaled as in Fig. 3, are constrained to saturate to an average of 2.0 at very high $\rho_{0}$. In essence, we get accurate measurements of the sample density and temperature in the exotic, intermediate regime of $\rho_{0}$, by assuming prior good understanding of behavior in the experimentally well-characterized regimes of low degeneracy, mean-field at high $T$ and of high-coherence, pure condensates at low $T$.

In Fig. 2, we present side-by-side pairs of coordinatespace and momentum-space distribution taken in a sequence such that pairs represent images of clouds with very similar temperatures and total atom number, such that the preimaging values of $\rho_{0}(0)$ are the same between pairs to within $10 \%$. As $T$ decreases, there is no obvious sudden change in the coordinate-space distribution $n_{0}(\vec{r})$, [Figs. 2(a)-(d)], while in momentum space [Figs. 2(e)-(h)] a central spike suddenly emerges at $T=171 \mathrm{nK}$ [Fig. 2(g)]. The inverse width of this central spike is a measure of the spatial extent of the coherent fraction in the highest-density region of the cloud. Our momentumspace resolution is such that the presence of a resolutionlimited peak implies that at least some coherence extends over a central disk of radius $4.5 \mu \mathrm{m}$, or $\gtrsim 10 \lambda_{d b}$. From coordinate-space images taken under the same conditions for which the coherence spike first appears in momentum space, we determine that it happens when the central value of $\rho_{0}=8.0(0.7)$ (this critical value $\rho_{c}$ is determined from looking at many more pairs of images than are presented in Fig. 2). We emphasize that from the coordinate-space distribution alone, the identification of a transition temperature would require model-dependent analysis of the smoothly varying distribution, while with access to both distributions at once, we readily see that 


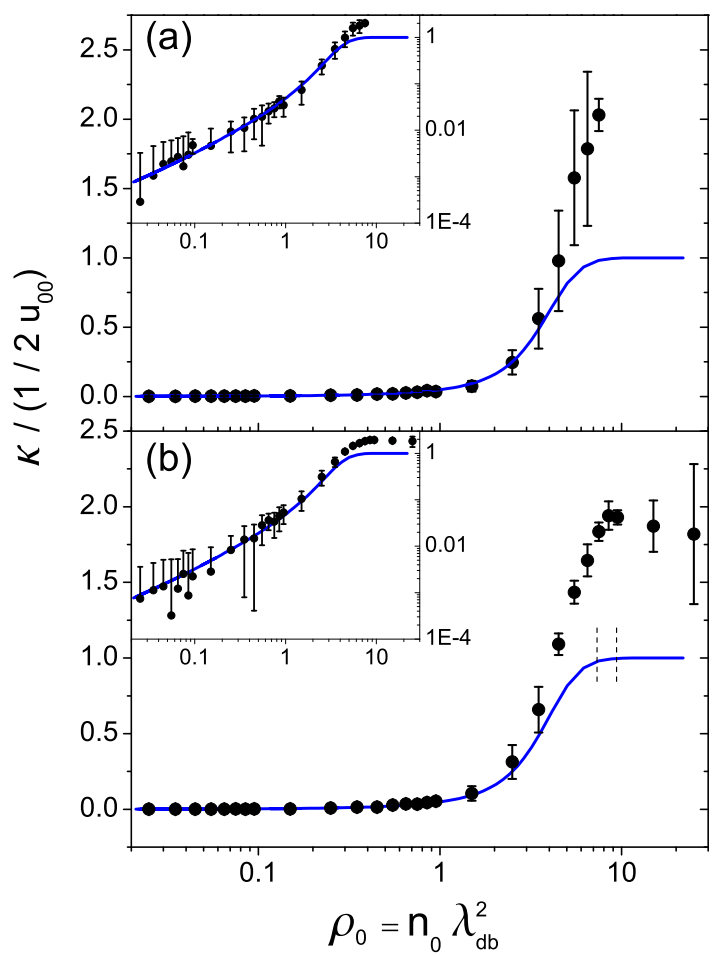

FIG. 3. Scaled compressibility $\kappa$ vs phase-space density $\rho_{0}$. (a) Measured $\kappa$ extracted from images of samples at $T=171$ $\mathrm{nK}$, as in the image in Fig.2(c). Black circles are data averaged over the values calculated from images of three separate clouds. The blue curves are $\kappa_{b a}$ calculated from the bareatom model. (b) Same but with $\kappa$ extracted from images of samples at $T=128 \mathrm{nK}$. The paired vertical dotted lines indicate the location of the jump in coherence discussed in the text.

a modest change in the central phase-space density of $<15 \%$ causes the distribution at $p=0$ to jump by a factor of 3 .

What have our observations told us about the 2D Bose gas as it cools towards the BKT transition? We can say empirically that as $\rho_{0}$ varies from about 7.2 to 8.7 , we see a dramatic increase of coherence in a range $>4.5$ $\mu \mathrm{m}$, jumping by a factor of 3 . The transition may be even sharper, but temporal drifts limit resolution. The predicted [14] critical value is $\rho_{c}=\ln (380 / \tilde{g})=8.3$.

Our most interesting observation is that in warmer gases, for $\rho_{0} \approx 4$, well before the sudden onset of coherence, we can already resolve that compressibility is above what a bare-atom model of degenerate bosons can account for. As $\rho_{0}$ reaches its coherence-jump value, 8.0, $\kappa / \kappa_{b a}$ has already increased to 1.7 (Fig. 3). It is natural to associate the increase in $\kappa / \kappa_{b a}$ with the gradual changing of the interaction energy from its value in a fully fluctuating gas, $2 u_{00} n_{0}$, to its fully condensed value, $u_{00} n_{0}$, and to draw a corresponding inference about the zerorange second-order correlation function. For $\tilde{g}=0.093$ the corresponding theoretical prediction (from Ref. 14] and Sec. 3.3 of Ref. [11]) for $\kappa / \kappa_{b a}$ at $\rho_{c}$ would be 1.59, in reasonable agreement with our observed 1.7. As a caveat, our observation of an anomalous $\kappa / \kappa_{b a}$ establishes definitively only the breakdown of the bare-atom model, which could be partially due to the violation of the other key bare-atom assumption, that in-plane excitations correspond to individual atoms with kinetic energy $\epsilon(\vec{p})=p^{2} / 2 m$. In any case, our data provide a first definitive observation of non-mea-nfield physics in the presuperfluid 2D Bose gas.

We are very pleased to acknowledge useful conversations with Z. Hadzibabic, J. Dalibard, W. Phillips, M. Holzmann, C. Chin, A. Imambekov, L.-K. Lim and V. Gurarie. This work was supported by NSF and ONR.

Note added.-Recently, a relevant experimental preprint appeared [19].

* Quantum Physics Division, National Institute of Standards and Technology, Boulder, CO, USA

[1] N. D. Mermin and H. Wagner, Phys. Rev. Lett. 17, 1133 (1966).

[2] J. M. Kosterlitz and D. J. Thouless, J. Phys. C 6, 1181 (1973); J. M. Kosterlitz, J. Phys. C 7, 1046 (1974); V. L. Berezinskii, Sov. Phys. JETP 32493 (1971); 34610 (1972).

[3] Z. Hadzibabic, P. Krüger, M. Cheneau, B. Battelier, and J. Dalibard, Nature (London) 441, 1118 (2006).

[4] P. Krüger, Z. Hadzibabic, and J. Dalibard, Phys. Rev. Lett. 99, 040402 (2007).

[5] S. P. Rath et al., Phys. Rev. A 82, 013609 (2010).

[6] P. Cladé, C. Ryu, A. Ramanathan, K. Helmerson, and W. D. Phillips, Phys. Rev. Lett. 102, 170401 (2009).

[7] V. Bagnato and D. Kleppner, Phys. Rev. A 44, 7439 (1991).

[8] D. S. Petrov, M. Holzmann, and G. V. Shlyapnikov, Phys. Rev. Lett. 84, 2551 (2000).

[9] Z. Hadzibabic, P. Krüger, M. Cheneau, S. P. Rath, and J. Dalibard, New J. of Phys. 10, 045006 (2008).

[10] V. Schweikhard, S. Tung, and E. A. Cornell, Phys. Rev. Lett. 99, 030401 (2007).

[11] Z.Hadzibabic and J. Daibard, arXiv:0912.1490v1.

[12] M. Holzmann, G. Baym, J.-P. Blaizot, and F. Laloë, Proc. Natl. Acad. Sci. U.S.A. 104, 1476 (2007).

[13] R. N. Bisset, M. J. Davis, T. P. Simula, and P. B. Blakie, Phys. Rev. A 79, 033626 (2009).

[14] N. Prokof'ev, O. Ruebenacker, and B. Svistunov, Phys. Rev. Lett. 87, 270402 (2001); N. Prokof'ev and B. Svistunov, Phys. Rev. A 66, 043608 (2002).

[15] M. Holzmann and W. Krauth, Phys. Rev. Lett. 100, 190402 (2008).

[16] c.f. $\tilde{g}=0.13[4]$ and $0.146[5]$ in Paris group. $\tilde{g}=0.02$ in Gaithersburg group [6].

[17] I. Shvarchuck et al., Phys. Rev. Lett. 89, 270404 (2002).

[18] A. H. van Amerongen et al., Phys. Rev. Lett. 100, 090402 (2008).

[19] C.-L. Hung, X. Zhang, N. Gemelke, and C. Chin, arXiv:1009.0016 